\title{
Species Specific Differences in the Ratio of Short to Long Loop Nephrons in the Kidneys of Laboratory Rodents
}

\author{
Osamu ICHII, Akira YABUKI, Toshimichi OJIMA, \\ Mitsuharu MATSUMOTO, and Shusaku SUZUKI \\ Laboratory of Veterinary Anatomy, Faculty of Agriculture, Kagoshima University, \\ 1-21-24 Korimoto, Kagoshima 890-0065, Japan
}

\begin{abstract}
The ratio of short to long loop nephrons (SLNs and LLNs, respectively) in laboratory rodents (mice, rats, hamsters, gerbils, and guinea pigs) was investigated using the air cast method. In mice and rats, the percentage of SLNs was significantly higher than that of LLNs, while in hamsters and gerbils, the reverse was true (\% of LLNs $>\%$ of SLNs). In guinea pigs, no significant difference in the percentages of LLNs and SLNs was noted.
\end{abstract}

Key words: kidneys of laboratory rodents, long loop nephrons, short loop nephrons

In mammalian kidneys, nephrons are divided into two types based on the length of the nephron loop. Short loop nephrons (SLNs) turn in the boundary between the outer medulla and inner medulla, while long loop nephrons (LLNs) turn in the inner medulla. Nephron loops are very important sites for the formation of the cortico-medullary osmotic gradient. Therefore, the ratio of SLNs to LLNs is an important index for evaluating the function of urine concentration in animal species. Previous studies have measured the ratio of SLNs to LLNs in several domestic and laboratory animals $[1,4-6,8,14]$. In laboratory rodents, assessment of this ratio in mice and rats has revealed that their major type of nephron is the SLN, not the LLN $[1,4,8$, 14]. Due to the technical difficulties associated with microdissection and micropuncture, these methods are not routinely used for measurement of the ratio of SLNs to LLNs.

Recently, we morphometricaly investigated the kidneys of laboratory rodents (mice, rats, hamsters, gerbils and guinea pigs), and identified species-dependent differences of kidney morphology [2]. However, species-dependent differences of the ratio of SLNs to LLNs among these animals remain unclear. The air cast method is a convenient method for visualization of nephrons $[11,12]$, and we used this technique to measure the ratio of SLNs to LLNs in mice, rats, hamsters, gerbils, and guinea pigs.

Male DBA/2CrSlc mice $(n=10)$, F344/NSlc rats $(\mathrm{n}=10)$, Slc:Syrian hamsters $(\mathrm{n}=10), \mathrm{MON} / \mathrm{JmsGbsSlc}$ gerbils $(n=5)$ and Slc:Hartley guinea pigs $(n=5)$ were purchased from SLC Japan (Shizuoka, Japan). All animals were housed in an open system room with a one-way airflow system (temperature $22 \pm 1{ }^{\circ} \mathrm{C}$; humid- 
ity $55 \pm 10 \%$; light period 07:00 to $19: 00$; ventilation 12 cycles/h), and were given ad libitum access to an autoclaved commercial diet (CE-2; Japan Clea, Tokyo, Japan) and tap water. The present study was performed in accordance with the Guidelines for Animal Experimentation of Kagoshima University. All animals were sacrificed at 3 months of age by exsanguination of the carotid arteries under deep anesthesia using a mixture of ketamine $(60 \mathrm{mg} / \mathrm{kg}$; Sankyo, Tokyo, Japan) and medetomidine (0.3 mg/kg; Meiji Seika, Tokyo, Japan). The kidneys were quickly removed and processed using a modification of the air cast method [11, 12]. Briefly, removed kidneys were fixed in $10 \%$ neutral buffered formalin, and sagittal sections $250 \mu \mathrm{m}$ thick including the renal papilla were cut using a microslicer (DTK-1000; Dosaka EM, Tokyo, Japan). The sections were dehydrated through a graded ethanol series and dried in liquid $\mathrm{CO}_{2}$ using a critical-point drier (HCP-2; Hitachi, Ibaraki, Japan). Dried sections were mounted on glass slides with xylene-dissoluted mounting medium (MGK-S; Matsunami, Osaka, Japan) and observed under a light microscope.

Figure 1 shows the morphometric scheme for measurement of the proportions of SLNs and LLNs. At the boundary between the outer and inner medulla, the numbers of turned loops (A) and unturned tubules (B) were counted. "A" was defined as the number of SLNs, and $\mathrm{B} \times 1 / 2$ was defined as the number of LLNs. Approximately 250 nephrons in each animal were measured, and the ratio of SLNs to LLNs was then calculated. The results are expressed as the mean \pm standard error (S.E.) and were analyzed statistically using the MannWhitney $\mathrm{U}$ test $(P<0.05)$.

Figure 2 shows representative light micrographs of kidney sections processed using the air cast method. This method clearly visualized SLNs turned loops and LLNs unturned tubules at the boundary between the outer and inner medulla in each species. Figure 3 summarizes the ratio of SLNs to LLNs in each species. In mice and rats, the percentage of SLNs was significantly higher than that of LLNs (SLN:LLN=64:36 and 57:43, respectively). In contrast, in hamsters and gerbils, the percentage of LLNs was significantly higher than that of SLNs (SLN:LLN=36:64 and 4:96, respectively). In guinea pigs, there was no significant difference in the percentages of SLNs and LLNs.

Previous studies using microdissection or micropunc-

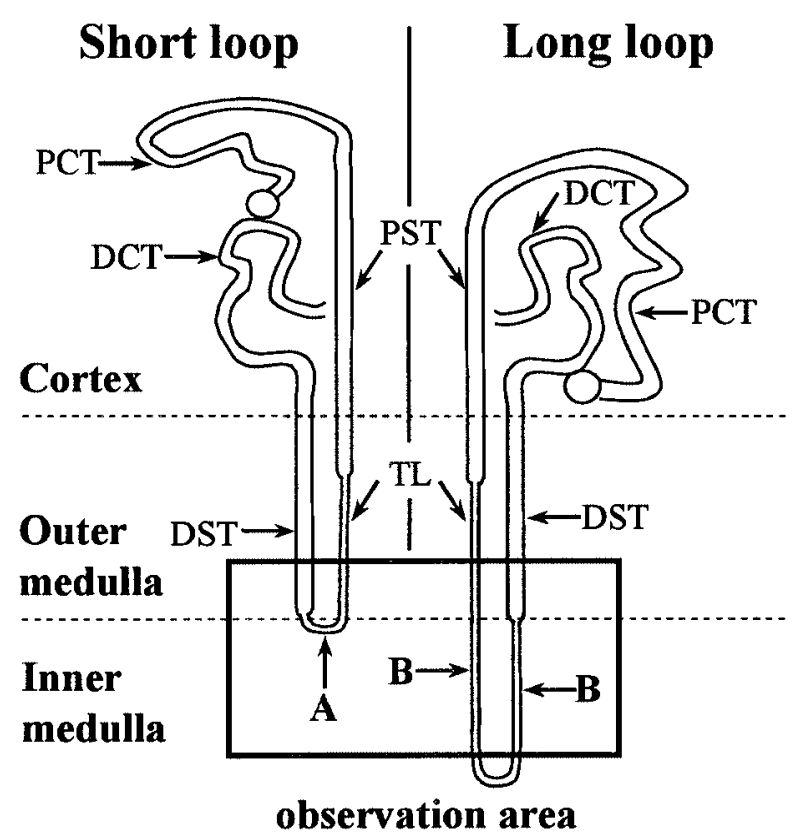

Fig. 1. Morphometric scheme for the measurement of the ratio of short to long loop nephrons (SLNs and LLNs, respectively). At the boundary between the outer medulla and inner medulla, the number of turned loops (A) and that of unturned tubules (B) were counted. The former was defined as the number of SLNs, and the latter $\times 1 / 2$ was defined as the number of LLNs. PCT: proximal convoluted tubule. PST: proximal straight tubule. TL: thin limb. DST: distal straight tubule. DCT: distal convoluted tubule.

ture clarified that mice and rats had higher percentages of SLNs [1, 7, 8, 14]. As a preliminary experiment, we prepared mouse kidney sections using the air cast method, and obtained results similar to those of the previous studies. Therefore, we used the air cast method to measure the ratio of SLNs to LLNs. In mice and rats, the percentage of SLNs was significantly higher than that of LLNs; however, the percentage of LLNs was higher than that of SLNs in hamsters and gerbils. Moreover, in guinea pigs, the percentages of SLNs and LLNs were not significantly different. From our present results using the air cast method, species-specific differences in nephron lengths were clarified in the kidneys of laboratory rodents.

Previous studies have demonstrated the important role of LLNs in urine concentration [3, 10, 14], as well as the relationship between the percentage of LLNs and urine concentration ability [13]. In the present study, although the percentages of LLNs in the rodent kidneys were in the order gerbil $(96 \%)>$ hamster $(64 \%)>$ 

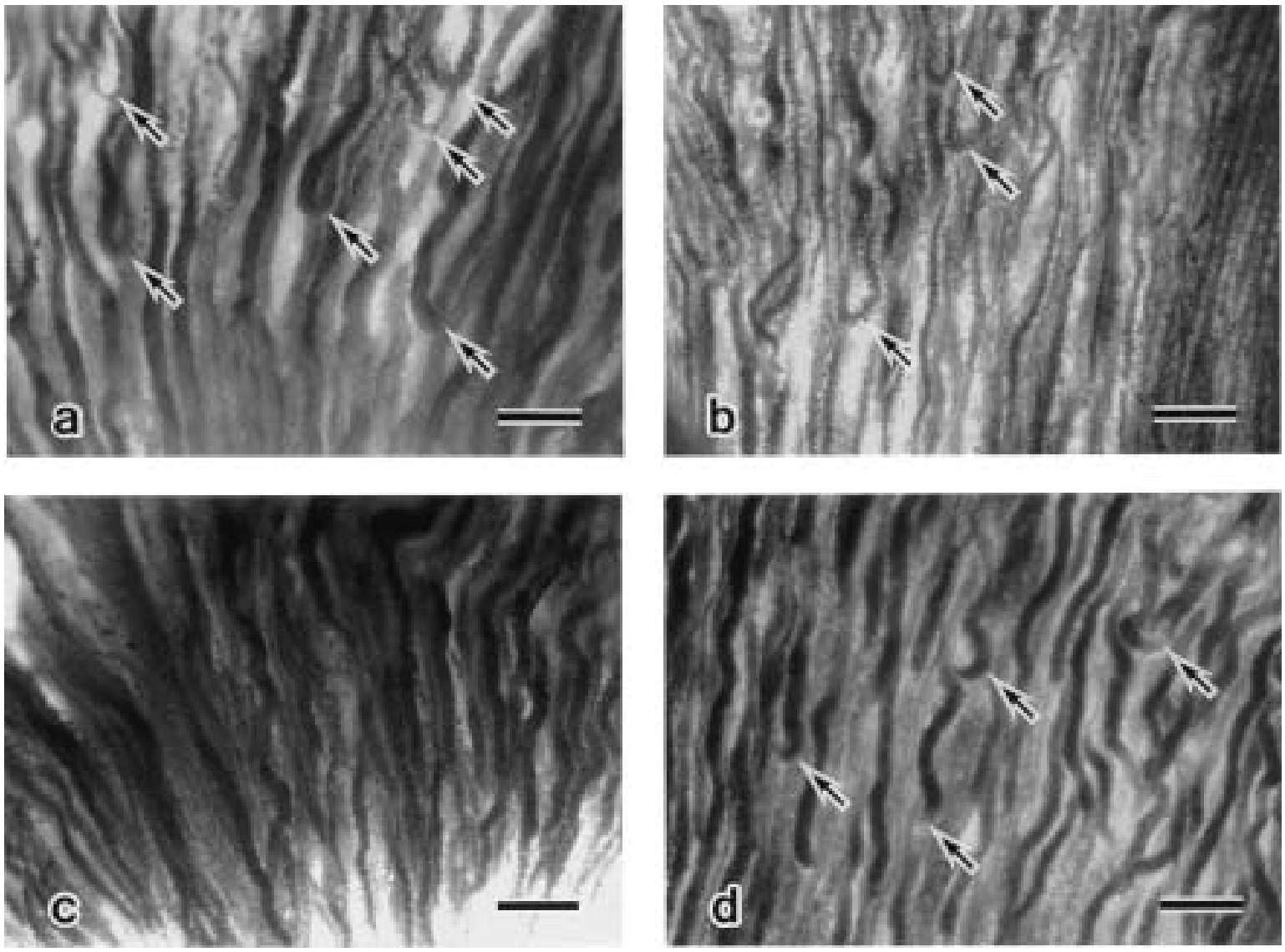

Fig. 2. The boundary between the outer and inner medulla in the kidneys of male mouse (a), hamster (b), gerbil (c), and guinea pig (d) processed using the air cast method. Turned loops of SLNs (arrows) and unturned tubules of LLNs are clearly visible. The number of SLN loops differs among species, being particularly numerous in the mouse, and rare in the gerbil. Bars: $50 \mu \mathrm{m}$.

guinea pig $(50 \%)>$ rat $(43 \%)>$ mouse $(36 \%)$, suitable correspondence between these species-specific differences and urine concentration ability, as reported by Munkacsi [9], was not shown. Briefly, in that report, maximum urine-concentrating capacity was measured as an index of urine concentration ability, with the order in laboratory rodents being gerbil $(5,400 \mathrm{mosm} / \mathrm{l})>$ hamster $(5,340 \mathrm{mosm} / \mathrm{l})>$ mouse $(4,300 \mathrm{mosm} / \mathrm{l})>$ rat $(3,250 \mathrm{mosm} / \mathrm{l})>$ guinea pig $(1,275 \mathrm{mosm} / \mathrm{l})$. Therefore, these results suggest that the species differences in urine concentration ability correlate with not only the ratio of SLNs to LLNs, but also with other indices, such as the ratio of juxtamedullary over superficial nephrons in the single-nephron glomerular filtration rate [1], glomerular volume [1] and proximal tubular length [1], ratio of medullary length to cortical thickness [9], and density of the vasa recta [9].
In conclusion, we determined the ratio of SLNs to LLNs in mice, rats, hamsters, gerbils and guinea pigs and clarified apparent species-specific differences in nephron loops in these laboratory rodents.

\section{References}

1. Bankir, L. and de Rouffignac, C. 1985. Am. J. Physiol. 249: 643-666.

2. Ichii, O., Yabuki, A., Ojima, T., Matsumoto, M., and Suzuki, S. 2006. J. Vet. Med. Sci. 68: 439-445.

3. Imai, M., Hayashi, M., and Araki, M. 1984. Pflugers. Arch. 402: 385-392.

4. Jamison, R. L. 1987. Kidney Int. 31: 597-605.

5. Kaissling, B. and Kriz, W. 1979. Adv. Anat. Embryol. Cell Biol. 56: 1-123.

6. Kaissling, B., de Rouffignac, C., Barrett, J. M., and Kriz, W. 1975. Anat. Embryol. 148: 121-143.

7. Kriz, W. 1967. Z. Zellforsch. Mikrosk. Anat. 82: 495-535.

8. Kriz, W. and Koepsell, H. 1974. Z. Anat. 

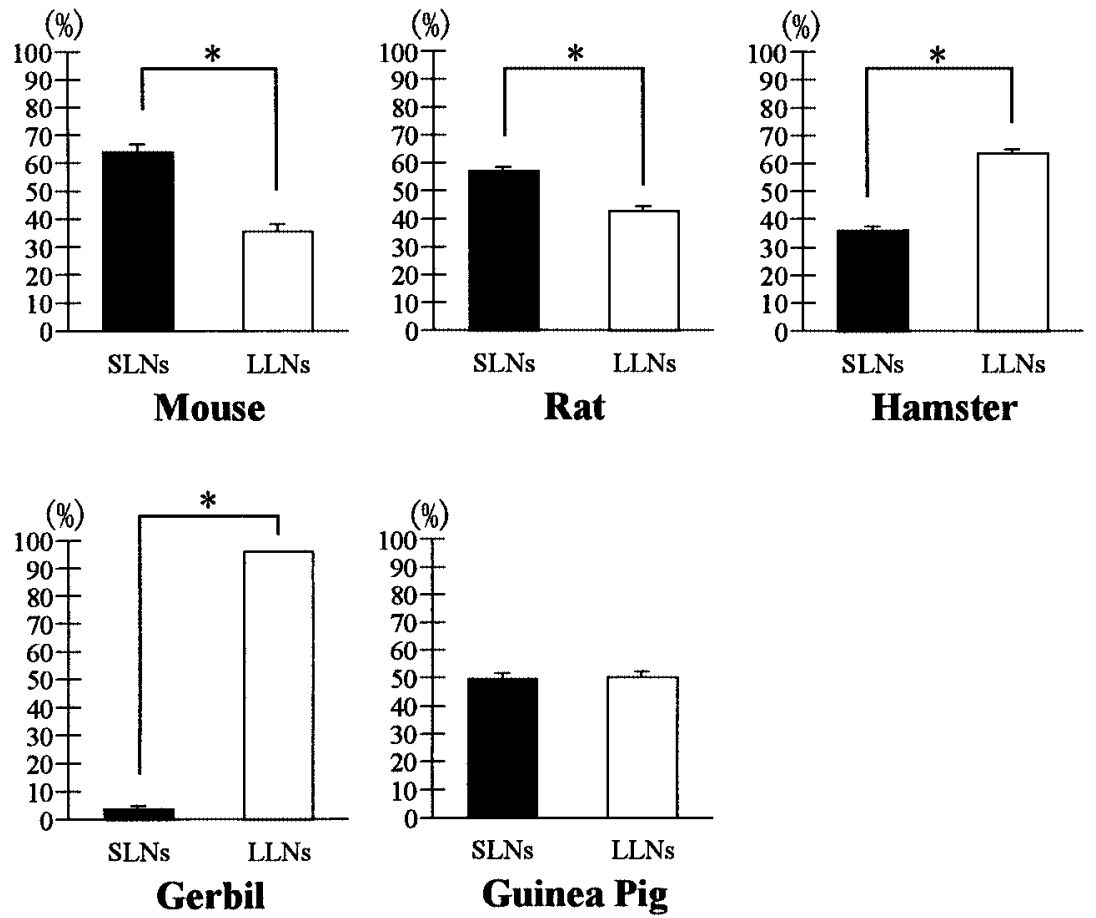

Fig. 3. The ratio of SLNs to LLNs for each animal. Each column represents the mean \pm S.E. *: Significant difference between SLNs and LLNs (Mann-Whitney U test, $P<0.05)$.

Entwicklungsgesch. 144: 137-163.

9. Munkacsi, I. and Palkovits, M. 1977. Acta. Anat. 98: 456468.

10. Nonoguchi, H., Tomita, K., and Marumo, F. 1992. J. Clin. Invest. 90: 349-357.

11. Ojeda, J. L., Garcia-Porrero, J. A., and Ros, M. A. 1984. Stain Technol. 59: 37-42.
12. Ojeda, J. L., Ros, M. A., and Garcia-Porrero, J. A. 1986. Acta. Anat. 126: 63-67.

13. Schmidt-Nielsen, B. and O'dell, R. 1961. Am. J. Physiol. 200: 1119-1124.

14. Sperber, I. 1944. Zoo. Bidrag. Fran. Uppsela. 22: 249437. 